\title{
An Improved Wavelet Approach for Finding Steady-State Waveforms of Power Electronics Circuits Using Discrete Convolution
}

\author{
Kam C. Tam, Student Member, IEEE, Siu-Chung Wong, Member, IEEE, and Chi K. Tse, Senior Member, IEEE
}

\begin{abstract}
Due to the switching action and the presence of parasitics, waveforms arising from power electronics circuits often contain high-frequency ringings embedded in slowly varying segments. Such a feature is consistent with the localization property of wavelets which has previously been exploited for fast approximations of steady-state waveforms. This paper proposes an improved and more robust approach for calculating the wavelet coefficients, exploiting the orthogonal property of the Chebyshev polynomials. Simulation results demonstrate the effectiveness of the new algorithm.
\end{abstract}

Index Terms-Chebyshev polynomials, power electronics circuits, steady-state solutions, wavelet transforms.

\section{INTRODUCTION}

$\mathbf{S}$ TEADY-STATE waveforms of power electronics circuits are important practical consideration for engineers, as they provide complete information on the operation of the circuits as well as the extent of current and voltage stresses on specific devices [1]-[3]. Obtaining the steady-state waveforms are, however, usually time consuming and may suffer from numerical instabilities, especially for power electronics circuits where slow and fast variations are present in different parts of the same waveform [3]. When describing waveforms arising from power electronics circuits, a frequently encountered problem is the fast variations near the switching instants. This usually appears as waveform ringings during the switching transitions and is caused by the presence of high-frequency resonant loops formed by small parasitic capacitances and inductances. Thus, the resulting waveforms contain largely low-frequency components with high-frequency details concentrated near the switching instants. Such a feature is consistent with the localization property of wavelets which are known in the signal processing literature to be highly suitable for describing fast changing edges embedded in slowly varying backgrounds [4]. It therefore appears that power electronics waveforms can be efficiently approximated by an appropriate set of wavelets.

Some preliminary work on the analysis of power electronics waveforms using wavelets was reported by Wernekinck, Valenzuela and Anfossi [5]. Recently, a systematic algorithm for ap-

Manuscript received April 27, 2004; revised October 29, 2004. This work was supported by the Hong Kong Polytechnic University under Grant A-PD68 and by the Hong Kong Research Grants Council under the CERG Grant. This paper was recommended by Associate Editor T. Ueta.

The authors are with the Department of Electronic and Information Engineering, Hong Kong Polytechnic University, Hong Kong (e-mail: encktse@polyu.edu.hk).

Digital Object Identifier 10.1109/TCSII.2005.852167 proximating steady-state waveforms arising from power electronics circuits using wavelets has been reported by Liu, Tse and $\mathrm{Wu}[6]$. The advantages of using wavelets for the analysis of power electronics circuits have also been demonstrated. The original algorithm has employed a standard curve-fitting method, which uses the boundary conditions together with extra interpolation points for solving the wavelet coefficients. The accuracy of the approximated solution is therefore dependent upon the positions of the interpolation points relative to the actual waveform to be found and limited by the wavelet levels used. It has been found that the solution is very sensitive to the choice of interpolation points for obtaining wavelet coefficients. A small mismatch between the set of interpolation points with the waveform to be approximated may lead to significant errors in the approximated solution, reducing the robustness of the method.

The aim of this paper is to tackle the limitations in the standard curve-fitting method. We will provide a new technique for obtaining better approximated waveforms with the same number of wavelet levels.

\section{Review of ORThogonality in Chebyshev Polynomials}

One important property of Chebyshev polynomials is that they are orthogonal in the sense that their inner product [7]-[9], defined by

$$
\left\langle T_{m}, T_{n}\right\rangle \stackrel{\text { def }}{=} \int_{-1}^{+1} \frac{T_{m}(x) T_{n}(x)}{\sqrt{1-x^{2}}} d x
$$

has the following property:

$$
\left\langle T_{m}, T_{n}\right\rangle= \begin{cases}0, & \text { if } m \neq n \\ \pi, & \text { if } m=n=0 \\ \frac{\pi}{2}, & \text { if } m=n \neq 0 .\end{cases}
$$

It is always possible to convert a (continuous) orthogonality relationship, as defined in (1), into a discrete orthogonality relationship simply by replacing the integral with a summation. In general, of course, the result is only approximately true. However, where trigonometric functions or Chebyshev polynomials are involved, there are many cases in which the discrete orthogonality can be shown to hold exactly, e.g.,

$$
\left\langle T_{m}, T_{n}\right\rangle \stackrel{\text { def }}{=} \sum_{k=1}^{i+1} T_{m}\left(x_{k}\right) T_{n}\left(x_{k}\right), \quad \text { for } 0 \leq m, n \leq i
$$

where $x_{k}$ are the zeros of $T_{i+1}(x)$. Here, we have

$$
x_{k}=\cos \theta_{k}, \theta_{k}=\frac{\left(k-\frac{1}{2}\right) \pi}{i+1}
$$


satisfying the orthogonality condition, i.e.,

$$
\left\langle T_{m}, T_{n}\right\rangle= \begin{cases}0, & \text { if } m \neq n ; m, n \leq i \\ (i+1), & \text { if } m=n=0 ; m \leq i \\ \frac{(i+1)}{2}, & \text { if } m=n \neq 0 ; m \leq i\end{cases}
$$

\section{WAVELET APPROXIMATION OF POWER ElECtronics WAVEFORMS USING DISCRETE CONVOLUTION}

It has been shown [6] that wavelet approximation can be useful for approximating steady-state solutions of nonlinear circuits without solving systems of nonlinear equations. In most cases, power electronics circuits can be represented by a time-varying state-space equation

$$
\dot{\boldsymbol{x}}=\boldsymbol{A x}+\boldsymbol{U}(t)
$$

where $\boldsymbol{x}$ is the $m$-dim state vector, $\boldsymbol{A}$ is an $m \times m$ time-varying matrix, and $\boldsymbol{U}$ is the input function. Specifically, we write

$$
\begin{aligned}
\boldsymbol{A}(t) & =\left[\begin{array}{cccc}
a_{11}(t) & a_{12}(t) & \cdots & a_{1 m}(t) \\
\vdots & \vdots & \ddots & \vdots \\
a_{m 1}(t) & a_{m 2}(t) & \cdots & a_{m m}(t)
\end{array}\right] \\
\boldsymbol{U}(t) & =\left[\begin{array}{c}
u_{1}(t) \\
\vdots \\
u_{m}(t)
\end{array}\right] .
\end{aligned}
$$

In the steady state, the solution satisfies

$$
x(t)=x(t+T), \quad \text { for } 0 \leq t \leq T
$$

where $T$ is the period. For an appropriate translation and scaling, the boundary condition can be mapped to the closed interval $[-1,1]$, i.e.,

$$
x(+1)=x(-1) .
$$

Assume that the basic time-invariant approximation equation is

$$
x_{i}(t)=\boldsymbol{K}_{i}^{T} \boldsymbol{\Psi}(t), \quad \text { for }-1 \leq t \leq 1 ; i=1,2, \cdots, m
$$

where $\Psi(t)$ is any wavelet basis of size $2^{n+1}+1$ ( $n$ being the wavelet level) constructed from Chebyshev polynomials, ${ }^{1}$ $\boldsymbol{K}_{i}^{T}=\left[k_{i, 0} \cdots k_{i, 2^{n+1}}\right]$ is a coefficient vector of dimension $2^{n+1}+1$, which is to be found.

The wavelet transformed equation of (6) is

$$
\boldsymbol{K D} \boldsymbol{\Psi}=\boldsymbol{A}(t) \boldsymbol{K} \boldsymbol{\Psi}+\boldsymbol{U}(t)
$$

${ }^{1}$ The construction of wavelet basis has been discussed in detail in Liu et al. where $D \Psi=(d / d t) \Psi($ see [6])

$$
\boldsymbol{K}=\left[\begin{array}{cccc}
k_{1,0} & k_{1,1} & \cdots & k_{1,2^{n+1}} \\
k_{2,0} & k_{2,1} & \cdots & k_{2,2^{n+1}} \\
\vdots & \vdots & \ddots & \vdots \\
k_{m, 0} & k_{m, 1} & \cdots & k_{m, 2^{n+1}}
\end{array}\right]
$$

Thus, (12) can be written generally as

$$
\boldsymbol{F}(t) \overline{\boldsymbol{K}}=-\boldsymbol{U}(t)
$$

where $\boldsymbol{F}(t)$ is a $m \times\left(2^{n+1}+1\right) m$ matrix, as given by the equation shown at bottom of page, and $\overline{\boldsymbol{K}}$ is a $\left(2^{n+1}+1\right) m$-dim vector given by

$$
\overline{\boldsymbol{K}}=\left[\begin{array}{lll}
\boldsymbol{K}_{1}^{T} & \cdots & \boldsymbol{K}_{m}^{T}
\end{array}\right]^{T} .
$$

Note that since the unknown $\bar{K}$ is of dimension $\left(2^{n+1}+1\right) m$, we need $\left(2^{n+1}+1\right) m$ equations. Now, the boundary condition (10) provides $m$ equations, i.e.,

$$
[\Psi(1)-\Psi(-1)]^{T} \boldsymbol{K}_{i}=\mathbf{0}, \quad \text { for } i=1, \cdots, m .
$$

In the original curve-fitting approach, $2^{n+1} m$ equations are obtained by interpolating at $2^{n+1}$ distinct points in the closed interval $[-1,1]$, and the interpolation points are chosen from the zeros of Chebyshev polynomials of the second kind, i.e.,

$$
\eta_{i}^{\left(2^{n+1}\right)}=\cos \left(\frac{i \pi}{2^{n+1}}\right) .
$$

It is obvious from (16) that one of the end points of either $\eta_{0}=1$ or $\eta_{2^{n+1}}=-1$ must be excluded from the interpolation points, as will be discussed in Section IV. In contrast, our new approach here takes advantage of the orthogonality in Chebyshev polynomials. The $2^{n+1} m$ more equations are obtained by taking discrete convolution over the closed interval $[-1,1]$ with $T_{i}$ for $i=0, \cdots, 2^{n+1}-1$. The number of points for discrete convolution is therefore no longer limited by the wavelet level in the calculation. Also, as discrete convolution is performed over individual wavelets, the relative weightings of the wavelet bases required become irrelevant and the exact locations of the data points are unimportant. Thus, this discrete convolution approach is expected to give a more robust and more accurate solution. In discrete convolution, the approximate solution is

$$
\tilde{\boldsymbol{F}} \overline{\boldsymbol{K}}=\tilde{\boldsymbol{U}}
$$

where

$$
\tilde{\boldsymbol{F}}=\left[\begin{array}{c}
\tilde{\boldsymbol{F}}_{1} \\
\tilde{\boldsymbol{F}}_{2}
\end{array}\right] \quad \tilde{\boldsymbol{U}}=\left[\begin{array}{c}
\tilde{\boldsymbol{U}}_{1} \\
\tilde{\boldsymbol{U}}_{2}
\end{array}\right]
$$

$$
\boldsymbol{F}(t)=\left[\begin{array}{ccccc}
a_{11}(t) \boldsymbol{\Psi}^{T}(t) \boldsymbol{D}^{T}-\boldsymbol{\Psi}^{T}(t) & \cdots & a_{1 i}(t) \boldsymbol{\Psi}^{T}(t) & \cdots & a_{1 m} \boldsymbol{\Psi}^{T}(t) \\
\vdots & \ddots & \vdots & \ddots & \vdots \\
a_{i 1}(t) \boldsymbol{\Psi}^{T}(t) & \cdots & a_{i i}(t) \boldsymbol{\Psi}^{T}(t)-\boldsymbol{\Psi}^{T}(t) \boldsymbol{D}^{T} & \cdots & a_{i m} \boldsymbol{\Psi}^{T}(t) \\
\vdots & \ddots & \vdots & \ddots & \vdots \\
a_{m 1}(t) \boldsymbol{\Psi}^{T}(t) & \cdots & a_{m i}(t) \Psi^{T}(t) & \cdots & a_{m m} \boldsymbol{\Psi}^{T}(t)-\Psi^{T}(t) \boldsymbol{D}^{T}
\end{array}\right]
$$


and $\tilde{\boldsymbol{F}}_{1}, \tilde{\boldsymbol{F}}_{2}, \tilde{\boldsymbol{U}}_{1}$, and $\tilde{\boldsymbol{U}}_{2}$ are given by the equation at bottom of page, and

$$
\begin{aligned}
& \tilde{\boldsymbol{F}}_{2}= {\left.\left[\begin{array}{c}
\left\langle\boldsymbol{F}(t), T_{0}(t)\right\rangle \\
\left\langle\boldsymbol{F}(t), T_{1}(t)\right\rangle \\
\vdots \\
\left\langle\boldsymbol{F}(t), T_{2^{n+1}-1}(t)\right\rangle \\
\left(2^{n+1}+1\right) m \text { columns }
\end{array}\right]\right\} 2^{n+1} m \text { rows } } \\
&\left.\tilde{\boldsymbol{U}}_{1}=\left[\begin{array}{c}
0 \\
\vdots \\
0
\end{array}\right]\right\} m \text { elements } \\
&\left.\tilde{\boldsymbol{U}}_{2}=\left[\begin{array}{c}
\left\langle-\boldsymbol{U}(t), T_{0}(t)\right\rangle \\
\left\langle-\boldsymbol{U}(t), T_{1}(t)\right\rangle \\
\vdots \\
\left\langle-\boldsymbol{U}(t), T_{2^{n+1}-1}(t)\right\rangle
\end{array}\right]\right\} 2^{n+1} m \text { elements }
\end{aligned}
$$

with $\left\langle\boldsymbol{F}(t), T_{k}(t)\right\rangle$ representing a new matrix whose elements are the corresponding convolutions with $T_{k}$. By solving (18), we obtain all the coefficients necessary for generating an approximate solution for the steady-state system.

\section{SimUlations AND EVALUATIONS}

The results are evaluated using the mean relative error (MRE) and mean absolute error (MAE), which are defined as

$$
\begin{aligned}
& \mathrm{MRE}=\frac{1}{2} \int_{-1}^{1}\left|\frac{\hat{x}(t)-x(t)}{x(t)}\right| d t \\
& \mathrm{MAE}=\frac{1}{2} \int_{-1}^{1}|\hat{x}(t)-x(t)| d t
\end{aligned}
$$

where $\hat{x}(t)$ is the wavelet-approximated value and $x(t)$ is the SPICE simulated result. The SPICE result, being generated from exact time-domain simulation of the actual circuit at device level, can be used for comparison and evaluation.

In discrete forms, MAE and MRE are simply given by

$$
\begin{aligned}
& \mathrm{MRE}=\frac{1}{N} \sum_{i=1}^{N}\left|\frac{\hat{x}_{i}-x_{i}}{x_{i}}\right| \\
& \mathrm{MAE}=\frac{1}{N} \sum_{i=1}^{N}\left|\hat{x}_{i}-x_{i}\right|
\end{aligned}
$$

where $N$ is the total number of points sampled along the interval $[-1,1]$ for error calculation. In the following, we use uniform sampling (i.e., equal spacing) with $N=1001$, including boundary points.

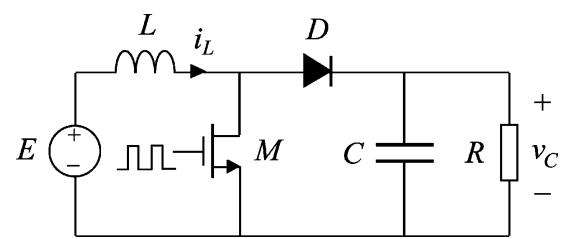

Fig. 1. Simple boost converter circuit.

TABLE I

COMPONENT AND PARAMETER VALUES FOR THE BOOST CONVERTER

\begin{tabular}{ll}
\hline Component/Parameter & Value \\
\hline Inductance, $L$ & $0.2 \mathrm{mH}$ \\
Capacitance, $C$ & $0.2 \mathrm{mF}$ \\
Load resistance, $R$ & $12.5 \Omega$ \\
Input voltage, $E$ & $16 \mathrm{~V}$ \\
Diode forward drop, $V_{f}$ & $0.8 \mathrm{~V}$ \\
Switching period, $T$ & $100 \mu \mathrm{s}$ \\
On-time, $T_{D}$ & $45 \mu \mathrm{s}$ \\
Switch on-resistance, $R_{S}$ & $0.001 \Omega$ \\
Diode on-resistance, $R_{D}$ & $0.001 \Omega$ \\
\hline
\end{tabular}

Example 1: Simple Boost Converter: We consider a simple boost converter, as shown in Fig. 1. Suppose the switch has a resistance of $R_{s}$ when it is turned on, and is practically open-circuited when it is turned off. The diode has a forward voltage drop of $V_{f}$ and an on-resistance of $R_{D}$. The basic system equation can be readily found as

$$
\dot{\boldsymbol{x}}=\boldsymbol{A}(t) \boldsymbol{x}+\boldsymbol{U}(t)
$$

where $\boldsymbol{x}=\left[i_{L} v_{C}\right]^{T}$, and $\boldsymbol{A}(t)$ and $\boldsymbol{U}(t)$ are given by

$$
\begin{aligned}
A(t) & =\left[\begin{array}{cc}
-\frac{R_{S}(1-s(t))+R_{D} s(t)}{L} & -\frac{s(t)}{L} \\
\frac{s(t)}{C} & -\frac{1}{R C}
\end{array}\right] \\
U(t) & =\left[\begin{array}{ll}
\frac{E-s(t) V_{f}}{L} & 0
\end{array}\right]^{T}
\end{aligned}
$$

with $s(t)$ defined by

$$
s(t)= \begin{cases}0, & \text { for } 0 \leq t \leq T_{D} \\ 1, & \text { for } T_{D} \leq t \leq T \\ s(t-T), & \text { for all } t>T\end{cases}
$$

The circuit parameters and component values used in the calculations and simulations are shown in Table I.

We compare the standard curve-fitting method and the proposed discrete convolution method. The approximated waveforms for the inductor current is shown in Fig. 2. By inspection, the discrete convolution method almost always gives more accurate waveforms than the standard curve-fitting method. In order to compare the results quantitatively, the values of the MREs are provided in Table II. Finally, we note that the inductor current waveform has been very well approximated by using only 33

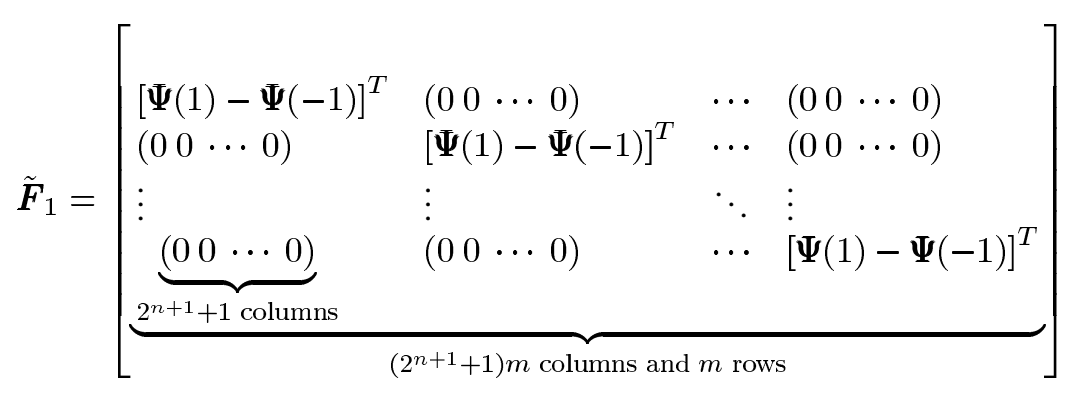


TABLE II

Comparison of MrEs for Approximating WaVeforms in Boost ConVerter. Data With * Denotes Poor Reference Provided by SPICE

\begin{tabular}{lccccc}
\hline Wavelet levels & Number of wavelets & $\begin{array}{c}\text { MRE for } i_{L} \\
\text { (curve fitting) }\end{array}$ & $\begin{array}{c}\text { MRE for } v_{C} \\
\text { (curve fitting) }\end{array}$ & $\begin{array}{c}\text { MRE for } i_{L} \\
\text { (proposed) }\end{array}$ & $\begin{array}{c}\text { MRE for } v_{C} \\
\text { (proposed) }\end{array}$ \\
\hline-1 to 4 & 33 & 0.112330 & 0.069052 & 0.025789 & 0.025714 \\
-1 to 5 & 65 & 0.068704 & 0.047254 & 0.025745 & 0.025704 \\
-1 to 6 & 129 & 0.047759 & 0.036652 & 0.025736 & 0.025703 \\
-1 to 7 & 257 & 0.036885 & 0.031222 & 0.025734 & 0.025702 \\
-1 to 8 & 513 & 0.031344 & 0.028473 & $0.031353^{*}$ & $0.028476^{*}$ \\
-1 to 9 & 1025 & 0.028547 & 0.027090 & not meaningful & not meaningful \\
-1 to 10 & 2049 & $0.030321^{*}$ & $0.028123^{*}$ & not meaningful & not meaningful \\
\hline
\end{tabular}

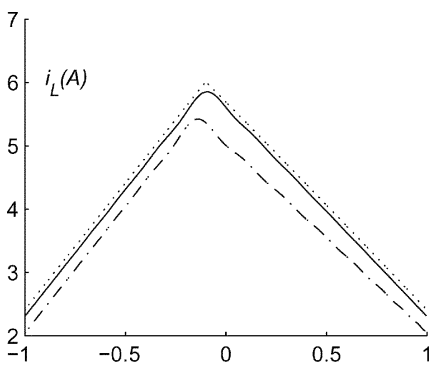

(a)

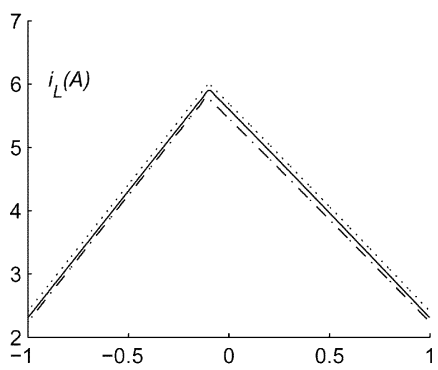

(c)

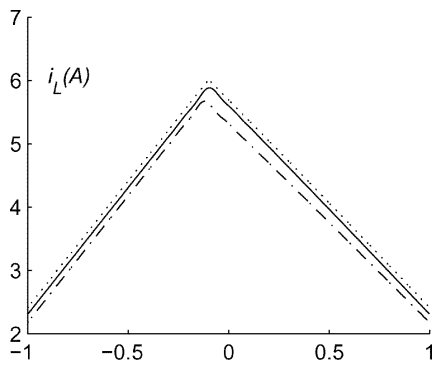

(b)

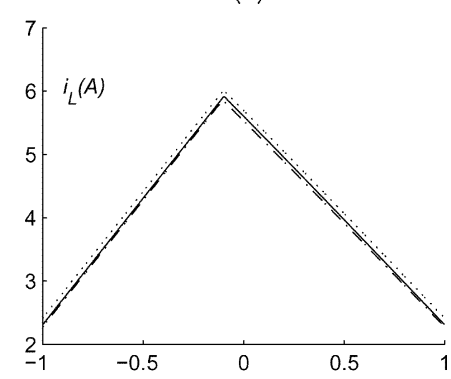

(d)

Fig. 2. Inductor current waveforms of boost converter. Solid line is wavelet-approximated waveform using discrete convolution, dotted line is waveform from SPICE simulation and dot-dashed line is waveform using curve-fitting method, (a) using wavelets of level -1 to 4 ; (b) using wavelets of level -1 to 5 ; (c) using wavelets of level -1 to 6 ; and (d) using wavelets of level -1 to 7 .

wavelets of level up to 4 in the proposed method with extremely small MREs.

It should be noted that the MRE generally decreases as more wavelet levels are used. Moreover, as evident from Table II, the trend appears to revert when wavelets up to a certain level are used, apparently implying that there may be an optimal wavelet level beyond which no further reduction in error can be expected. However, upon close inspection, the apparent increase in MRE when wavelet levels up to 8 (for the proposed method) and 10 (for the curve fitting method) are used is actually due to the limited accuracy of the SPICE reference waveforms [10]. In other words, SPICE results have become less accurate and hence their use as reference have deceptively given a larger MRE. Thus, data corresponding to any higher wavelet levels are not meaningful.

Example 2: Flyback Converter With Ringings: We now consider the flyback converter shown in Fig. 3(a). This is a more realistic model as the parasitic capacitance across the switch and leakage inductance of the transformer are deliberately included. The operation is as follows. When the switch is turned on, current flows through the magnetizing inductance

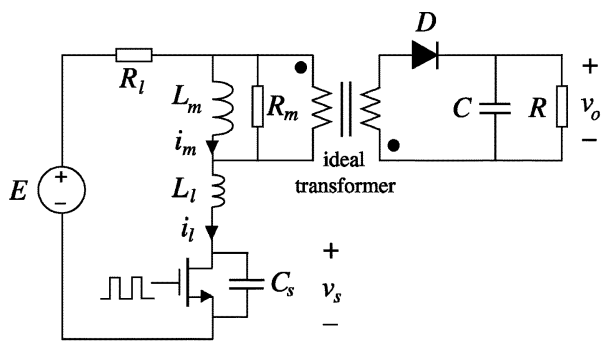

(a)

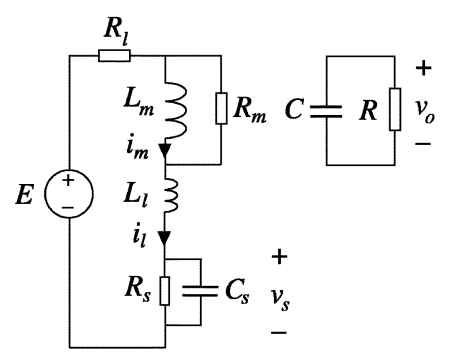

(b)

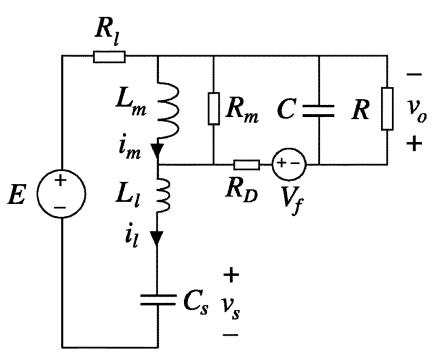

(c)
Fig. 3. (a) Flyback converter model with transformer leakage inductance and parasitic capacitance. (b) On-time circuit models. (c) Off-time circuit models.

$L_{m}$ and the leakage inductance $L_{l}$, with the transformer secondary opened and the diode not conducting. When the switch is turned off, the transformer secondary conducts through the diode, clamping the primary voltage (i.e., voltage across $L_{m}$ ) to the output network (assuming a 1:1 turns ratio). Thus, $L_{m}$ discharges through the transformer primary, while the leakage $L_{l}$ and the parasitic capacitance $C_{s}$ form a damped resonant loop around the input voltage source. Figs. 3(b) and (c) show the detailed circuit models for the on-time and off-time durations.

The state equation of this converter is given by

$$
\dot{\boldsymbol{x}}=\boldsymbol{A}(t) \boldsymbol{x}+\boldsymbol{U}(t)
$$

where $\boldsymbol{x}=\left[\begin{array}{llll}i_{m} & i_{l} & v_{s} & v_{o}\end{array}\right]^{T}$, and $\boldsymbol{A}(t)$ and $\boldsymbol{U}(t)$ are given by

$$
\begin{aligned}
& \boldsymbol{A}(t)=\boldsymbol{A}_{1}(1-s(t))+\boldsymbol{A}_{2} s(t) \\
& \boldsymbol{U}(t)=\boldsymbol{U}_{1}(1-s(t))+\boldsymbol{U}_{2} s(t)
\end{aligned}
$$

with $s(t)$ defined earlier in (27) and the $U^{\prime}$ 's and $\boldsymbol{A}$ 's being derivable from the circuit topologies.

The parameters for simulation are listed in Table III. We have compared the approximated waveforms of the leakage inductor current for both discrete convolution and curve-fitting method. Fig. 4(a) and (b) shows the approximated waveforms using discrete convolution at two different wavelet levels. Fig. 4(c) and (d) shows the approximated waveforms using the curve-fitting approach, excluding $\eta_{2^{n+1}}(=-1)$ and $\eta_{0}(=1)$, respectively. 
TABLE III

COMPONENT AND PARAMETER VALUES FOR FLYBACK CONVERTER

\begin{tabular}{ll}
\hline Component/Parameter & Value \\
\hline Magnetizing (storage) inductance, $L_{m}$ & $0.4 \mathrm{mH}$ \\
Leakage inductance, $L_{l}$ & $1 \mu \mathrm{H}$ \\
Equivalent parallel resistance of transformer primary, $R_{m}$ & $1 \mathrm{M} \Omega$ \\
Output capacitance, $C$ & $0.1 \mathrm{mF}$ \\
Load resistance, $R$ & $12.5 \Omega$ \\
Input voltage, $E$ & $16 \mathrm{~V}$ \\
Diode forward drop, $V_{f}$ & $0.8 \mathrm{~V}$ \\
Switching period, $T$ & $100 \mu \mathrm{s}$ \\
On-time, $T_{D}$ & $45 \mu \mathrm{s}$ \\
Equivalent loop resistance, $R_{l}$ & $0.4 \Omega$ \\
Switch on-resistance, $R_{S}$ & $0.001 \Omega$ \\
Switch capacitance, $C_{S}$ & $200 \mathrm{nF}$ \\
Diode on-resistance, $R_{D}$ & $0.001 \Omega$ \\
\hline
\end{tabular}

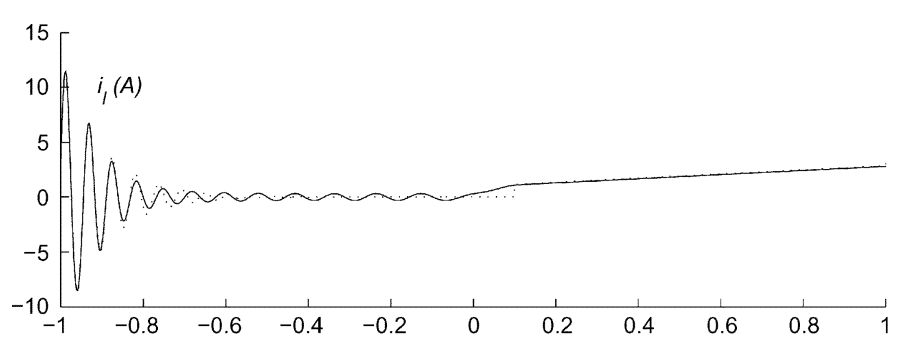

(a)

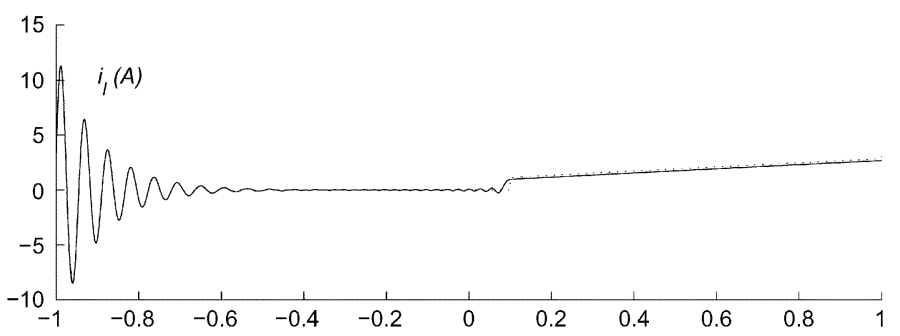

(b)

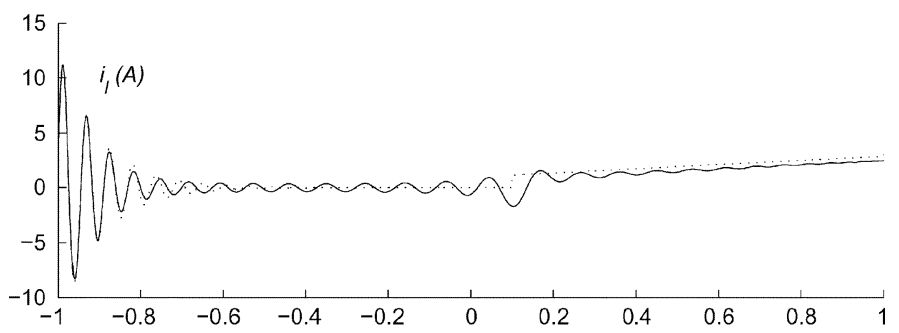

(c)

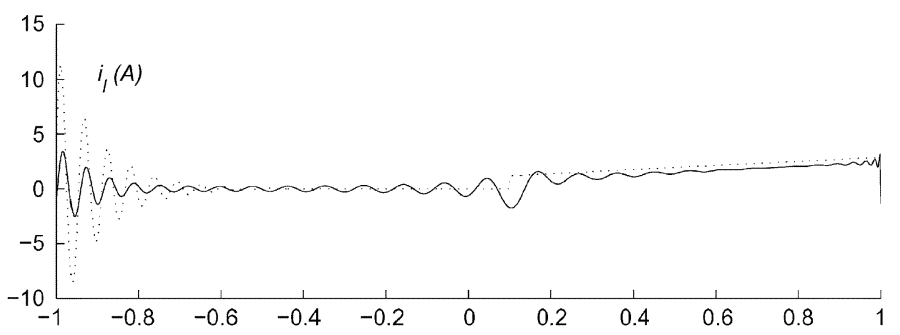

(d)

Fig. 4. Inductor waveforms of flyback converter. Dashed line is waveform from SPICE simulation. Solid line is approximated waveform. (a) Using discrete convolution wavelets of level -1 to 5 . (b) Using discrete convolution wavelets of level -1 to 7 . (c) Using curve-fitting wavelets of level -1 to 5 excluding the interpolation point $\eta_{2 n+1}(=-1)$. (d) Using curve-fitting wavelets of level -1 to 5 excluding the interpolation point $\eta_{0}(=1)$.

As there is no systematic method to predict which exclusion would produce better result, the original curve-fitting method is less robust.
TABLE IV

COMPARISON OF MAEs FOR APPROXIMATING LEAKAGE INDUCTOR CURRENT IN Flyback CONVERTER. DATA WiTH * DENOTES POOR REFERENCE PROVIDED BY SPICE

\begin{tabular}{lccc}
\hline Wavelet levels & Number of wavelets & $\begin{array}{c}\text { MAE for } i_{l} \\
\text { (curve fitting) }\end{array}$ & $\begin{array}{c}\text { MAE for } i_{l} \\
\text { (proposed) }\end{array}$ \\
\hline-1 to 5 & 65 & 0.60376 & 0.24045 \\
-1 to 6 & 129 & 0.52070 & 0.15298 \\
-1 to 7 & 257 & 0.36704 & 0.15314 \\
-1 to 8 & 513 & 0.18808 & $0.16106^{*}$ \\
\hline
\end{tabular}

Since the waveform contains a substantial portion where the value is near zero, we use the MAE as the evaluation metric. Better results from the original method are selected for comparison in Table IV which clearly verifies the advantage of using discrete convolution for wavelet approximation. The problem of SPICE's failing to provide an accurate reference is also apparent here.

As a final remark on the computational speed, we generally observe a 10 to 20 times reduction in the computation time offered by the proposed method over the curve fitting method. For example, to achieve an MAE of less than 0.18 for $i_{l}$, the proposed method takes $14.9 \mathrm{~s}$ on a Pentium III machine, whereas the curve fitting method takes $265.2 \mathrm{~s}$. To achieve an MAE of less than 0.37 , the proposed method and the curve fitting method take 2.516 and $31.98 \mathrm{~s}$, respectively.

\section{CONCLUSION}

In this paper, we propose a robust algorithm for approximating waveforms of power electronics using wavelets. The algorithm exploits the orthogonality property to provide the necessary alternative equations for solving the wavelet coefficients. Furthermore, it has been shown that the discrete convolution method offers good robustness as well as numerical accuracy over the original curve-fitting approach.

\section{REFERENCES}

[1] D. Li and R. Tymerski, "A comparison of steady-state methods for power electronic circuits," in IEEE Power Electronics Specialists Conf. Rec., vol. 2, 1998, pp. 1084-1090.

[2] P. T. Krein, Elements of Power Electronics. New York: Oxford University Press, 1998.

[3] Y. S. Lee, Computer-Aided Analysis and Design of Switch Mode Power Supplies. New York: Marcel Dekker, 1993.

[4] I. Daubechies, "Time-frequency localization operators: a geometric phase space approach," IEEE Trans. Inf. Theory, vol. 34, pp. 605-612, 1988.

[5] E. Wernekinck, H. Valenzuela, and I. Anfossi, "On the analysis of power electronics circuits waveforms with wavelets," in Proc. Power Conversion Conf., 1993, pp. 544-549.

[6] M. Liu, C. K. Tse, and J. Wu, "A wavelet approach to fast approximation of steady-state waveforms of power electronics circuits," Int. J. Circuit Theory Appl., vol. 31, no. 6, pp. 591-610, Nov. 2003.

[7] J. C. Mason and D. C. Handscomb, Chebyshev Polynomials. Boca Raton, FL: Chapman and Hall/CRC, 2003.

[8] T. Kilgore and J. Prestin, "Polynomial wavelets on the interval," Constr. Approx., vol. 12, pp. 95-110, 1995.

[9] B. Fisher and J. Prestin, "Wavelets based on orthogonal polynomials," Math. Comput., vol. 66, no. 220, pp. 1593-1618, 1997.

[10] L. O. Chua and P. M. Lin, Computer-Aided Analysis of Electronic Circuits. Englewood Cliffs, NJ: Prentice-Hall, 1975. 